$13^{\text {th }}$ International Conference on

AEROSPACE SCIENCES \& AVIATION TECHNOLOGY,

$\boldsymbol{A S A T - 1 3 ,}$ May 26 - 28, 2009, E-Mail: asat@mtc.edu.eg

Military Technical College, Kobry Elkobbah, Cairo, Egypt

Tel : +(202) 24025292-24036138, Fax: +(202) 22621908

\title{
Performance Analysis of DS-CDMA Receiver in Presence of Partial Band Jamming
}

\author{
Ahmed El-Mahdy ${ }^{*}$, Hossam E. Abou-Bakr ${ }^{*}$ and Yasser Mahmoud ${ }^{*}$
}

\begin{abstract}
Direct Sequence Code Division Multiple Access (DS-CDMA) is a multiple access technique that differentiates between different users by assigning unique spreading codes to them. It is widely used in communication systems because of its great bandwidth efficiency and its multiple access capabilities. DS-CDMA signals are resistant to multipath fading. Since the spread spectrum signal occupies a large bandwidth, only a small portion of this signal will undergo fading due to multipath at any given time. Moreover, DS-CDMA can also effectively reject narrowband interference, since narrowband interference affects only a small portion of the spread spectrum signal. In this paper, the performance of a DS-CDMA system is investigated in presence of multiple-access interference (MAI) and an additive white Gaussian noise (AWGN) under the presence of partial band jamming. Two types of receivers are proposed, single user matched filter receiver (SUMF) and decorrelator receiver.
\end{abstract}

Keywords: DS-CDMA, SUMF, AWGN, partial band jamming

\section{Introduction}

There are many techniques used in communication systems to access multiple users such as Time Division Multiple Access (TDMA) where each user is assigned to a specific time slot, Frequency Division Multiple Access (FDMA) where each user is assigned to a specific frequency band and Direct Sequence Code Division Multiple Access (DS-CDMA) where a specific code is assigned to each user over the same time interval and the same bandwidth. In DS-CDMA systems, the signal power is spread over a large bandwidth. Thus, it will be too difficult to be detected and intercepted by a hostile receiver. Another interesting feature of CDMA is its anti-jamming capability which means that a narrowband jamming signal is spreaded at the receiver input so that the received jamming signal is reduced by a factor equal to the processing gain [3].

The performance of the DS-CDMA system in the presence of jamming is analyzed in several articles [1] [2]. In [1], the performance of MC-CDMA in the presence of a narrowband jamming is analyzed. In [2], the performance of a CDMA communication system in the presence of a partial-band jamming is presented where the effects of multipath and AWGN are analyzed. The above articles are concerned with improving the receiver ability and are not concerned with improving the jamming ability. In this paper, the effect of a partial band jamming on the performance of DS-CDMA systems is evaluated. The effect of a partial band jamming is analyzed in Binary Phase Shift Keying (BPSK) system. It is assumed that there are coherent detection and perfect symbol synchronization in an AWGN channel.

\footnotetext{
* Egyptian Armed Forces
} 
The remainder of this paper is organized as follows: In Section II, a system model for DSCDMA system under the jamming environment is presented. In Section III, the performance of both SUMF and decorrelator receivers under partial band jamming is evaluated. Section $\mathrm{IV}$, the numerical results and computer simulations are presented to verify the theoretical expressions proved in section III. Finally, the conclusions are provided in Section V.

\section{System Model}

A partial-band noise jammer spreads Gaussian noise of total power J evenly over a part of the total frequency range of the spreading bandwidth. A model of the CDMA system under jamming environment is described in [7] and is shown in Fig. 1. It is assumed that there are $\mathrm{K}$ active users transmission. The received signal during one symbol interval, $T_{b}$, has the following form:

$r(t)=\sum_{k=1}^{K} \sqrt{2 P} C_{k}(t) b_{k}(t) \cos \omega_{c} t+j(t)+n(t)$

where $b_{k}(t) \in\{-1,+1\}$ is the data symbol of the $k^{t h}$ user with equal probabilities to be 1 or $-1, P$ is the received power of $k^{t h}$ user (assuming that all users transmit the same power), $n(t)$ is an AWGN with two-sided power spectral density equal to $N_{o} / 2, \omega_{c}$ is the carrier frequency and $j(t)$ is the jamming signal which is modeled as AWGN with two-sided power spectral density has the value of $N_{j} / 2 \rho$, where $\rho$ is the partial band factor of the jamming signal. In (1) $C_{k}$ is the signature sequence of +1 's and -1 's assigned to the $K^{\text {th }}$ user and it is given by

$C_{k}(t)=\sum_{i=0}^{N} C_{k} \psi\left(t-i T_{c}\right)$

where $\psi(t)$ is the unit rectangular pulse of duration $T_{c}=T_{b} / N$ and $N$ is the number of bits in the spreading sequence.

\section{Performance Analysis of DS-CDMA Systems under Partial Band Jamming}

\section{A. SUMF Receiver}

The output of the matched filter is a dispread and demodulated signal. The dispread signal is obtained by applying the appropriate PN sequence [3]. The demodulated signal is simply obtained by applying a modulating tone to provide a coherent detection. The output of the receiver can be written as

$$
\begin{aligned}
Z_{k} & =\int_{0}^{T_{b}} r(t) C_{k}(t) \cos \left(\omega_{c} t\right) d t \\
& =B_{k}+I+\eta+J
\end{aligned}
$$

where $B_{k}$ is the information of the aimed user, $I$ is the multiple access interference, $\eta$ is an AWGN, $J$ is the partial band jamming interference. The output of the matched filter consists of the following: 
1- The aimed user information $B_{k}$ :

$$
\begin{aligned}
B_{k} & =\int_{0}^{T_{b}} \sqrt{2 P} C_{k}^{2}(t) b_{k}(t) \cos 2 \omega_{c} t d t \\
& = \pm \sqrt{\frac{P}{2}} T_{b}
\end{aligned}
$$

where $\cos ^{2} \omega_{c} t=\frac{1}{2}\left(1+2 \omega_{c} t\right), C_{k}^{2}(t)=1$. Since the multiplication of $\omega_{c}$ and $t$ was selected as the integer number, the integration of $\cos \omega_{c} t$ and $\sin \omega_{c} t$ functions over one period is always zero. Likewise the integration of $b_{k}(t)$ over one period is 1 because the number of 1 's and -1 's is assumed to be the same.

2- The multiple access interference I which is represented as:

$$
\begin{aligned}
I & =\int_{0}^{T_{b}} \sum_{i=1, i \neq k}^{K} \sqrt{2 P} C_{k}(t) C_{i}(t) b_{k}(t) \cos 2 \omega_{c} t \\
& = \pm \sum_{i=1, i \neq k}^{K} \sqrt{\frac{P}{2}} \rho_{i k} T_{b}
\end{aligned}
$$

where $\rho_{i k}=\int_{0}^{T_{b}} C_{i}(t) C_{k}(t) d t$ represents the cross-correlation function of the spreading codes.

3 - The AWGN power $\eta$ is given by:

$\eta=\int_{0}^{T_{b}} n(t) C_{k}(t) \cos \omega_{c} t d t$

The partial band jamming interference Jis given by:

$J=\int_{0}^{T_{b}} j(t) C_{k}(t) \cos \omega_{c} t d t$

The decision statistic of the output of the matched filter, $Z_{k}$, can be modeled as Gaussian random variable with a mean value of $\bar{Z}$ and variance of $\sigma_{Z}^{2}$ where the mean value of the decision statistics $Z$ is

$E\{Z\}=\bar{Z}=E\left\{B_{k}+I+\eta+J\right\}$

$\bar{Z}=E\left\{B_{k}\right\}+E\{I\}+E\{\eta\}+E\{J\}$

where $E\left\{B_{k}\right\}= \pm \sqrt{\frac{P}{2}} T_{b}, E\{I\}$ is the multiple access interference term mean that is defined in [1] as zero mean $E\{I\}=0[1], E\{\eta\}$ is the noise power mean which equal to zero $E\{\eta\}=0$ as $n(t)$ is a zero mean AWGN, the jamming interference mean which equals zero $E\{J\}=0$ since $j(t)$ is a zero mean Gaussian random variable.

The variance of $Z, \sigma_{Z}^{2}$, is the sum of the variances of the multiple access interference term and noise plus jamming term, which are assumed to be independent. Therefore, $\sigma_{Z}^{2}$ can be written as:

$\sigma_{Z}^{2}=\sigma_{I}^{2}+\sigma_{\eta}^{2}+\sigma_{J}^{2}$ 
The variance of multiple access interference term, $\sigma_{I}^{2}$, is defined in [3] as:

$\sigma_{I}^{2}=T_{c}^{2}(k-1) P N / 6$

and the variance of noise term, $\sigma_{\eta}^{2}$, is

$$
\begin{aligned}
\sigma_{\eta}^{2} & =E\left\{\eta^{2}\right\}-E\{\eta\}^{2} \\
& =E \int_{0}^{T_{b}} n(t) C_{k}(t) \cos \omega_{c} t d t \int_{0}^{T_{b}} n(\lambda) C_{k}(\lambda) \cos \omega_{c} \lambda d \lambda \\
& =E \int_{0}^{T_{b}} \int_{0}^{T_{b}} n(t) n(\lambda) C_{k}(t) C_{k}(\lambda) \cos \omega_{c} t \cos \omega_{c} \lambda d t d \lambda
\end{aligned}
$$

Assuming that $n(t)$ is a wide-sense stationary random process. Thus the autocorrelation of the white Gaussian random process was defined in [6] as:

$E\{n(t) n(\lambda)\}=\frac{N_{o}}{2} \delta(t-\lambda)$

So,

$$
\begin{aligned}
\sigma_{\eta}^{2} & =\frac{N_{o}}{2} \int_{0}^{T_{b}} C_{k}^{2}(t) \frac{1}{2}\left(1+\cos 2 \omega_{c} t\right) d t \\
& =\frac{N_{o} T_{b}}{4}
\end{aligned}
$$

For a jamming interference term it is assumed that $j(t)$ is a wide-sense stationary random process. The same procedures are applied to drive the variance of $J$ which is

$\sigma_{J}^{2}=\frac{N_{j} T}{4 \rho}$

The bit error probability for this system, $P_{e}$, is defined according to the Gaussian approximation [1], [6], as

$P_{e}=Q\left(\sqrt{\frac{\bar{Z}^{2}}{\sigma_{Z}^{2}}}\right)=Q\left(\sqrt{\frac{\left(\sqrt{\frac{P}{2}} T_{b}\right)^{2}}{\frac{T_{C}^{2} N}{6}(k-1) P+\frac{N_{o} T_{b}}{4}+\frac{N_{j} T_{b}}{4 \rho}}}\right)=Q\left[\left(\frac{(K-1)}{3 N}+\frac{N_{o}}{2 E_{b}}+\frac{N_{j}}{2 \rho E_{b}}\right)^{-1}\right]$

where $Q(x)=\frac{1}{\sqrt{2 \pi}} \int_{x}^{\infty} e^{\left(-\frac{u^{2}}{2}\right)} d u$ is the standard $Q$ function, $T_{c} N=T_{b}, T_{b} P=E_{b}$, where $E_{b}$ is the energy per bit.

\section{B. Decorrelator Receiver}

The received signal at the input of the decorrelator receiver during one bit interval, $T_{b}$, has the following form

$r(t)=\sum_{k=1}^{K} \sqrt{2 P} C_{k}(t) b_{k} \cos \omega_{c} t+j(t)+n(t)$

The output of the $k^{\text {th }}$ matched filter is given by 
$y_{k}=\int_{0}^{T_{b}} r(t) C_{k}(t) \cos \omega_{c} t d t$

which could be rewritten as:

$$
\begin{aligned}
& y_{1}=\sum_{i} A b_{k} R_{1 i}+n+j \\
& y_{2}=\sum_{i} A b_{k} R_{2 i}+n+j \\
& \dot{y} \\
& y_{K}=\sum_{i} A b_{K} R_{K i}+n+j
\end{aligned}
$$

The above equation could be written in vector representation as follows

$\boldsymbol{y}=\boldsymbol{R A b}+\boldsymbol{n}+\boldsymbol{j}$

where $\boldsymbol{A}$ is a diagonal matrix with a vector $\left[A_{1}, A_{2}, \ldots, A_{K}\right]^{T}$ represents it is main diagonal, $A_{k}=\sqrt{\frac{P}{2}} T_{b}, 1 \leq k \leq K$ assuming a constant power for all users, $\boldsymbol{b}=\left[b_{1}, b_{2}, \ldots, b_{K}\right]$ are the data bits from the $k$ users, $\boldsymbol{n}=\left[n_{1}, n_{2}, \ldots, n_{k}\right]$ is a vector of the Gaussian noise samples with zero mean and a covariance matrix $\sum n=\sigma_{n}^{2} \boldsymbol{R}$ (where $\sigma_{n}^{2}=N_{o} T_{b} / 4$ is the noise power after dispreading), and $\boldsymbol{j}=\left[j_{1}, j_{2}, \ldots, j_{K}\right]$ is a vector of partial band jamming signal which is modeled as Gaussian random process with zero mean and a covariance matrix $\sum j=$ $\sigma_{j}^{2} \boldsymbol{R}$ (where $\sigma_{j}^{2}=N_{o} T_{b} / 4 \rho$ is the jamming power after despreading), $\boldsymbol{R}$ is the non-negative definite matrix of the cross-correlations between the waveforms of the different users that's given by

$R_{i j}=\int_{0}^{T_{b}} c_{i}(t) c_{j}(t) d t$

The estimated value of the transmitted bit is given by

$\widehat{\boldsymbol{b}}=\operatorname{sign} \boldsymbol{R}^{-\mathbf{1}} \boldsymbol{y}$

The decision matrix $\mathbf{z}$ is equal to $\boldsymbol{R}^{-\mathbf{1}} \boldsymbol{y}$, and the covariance matrix $\Sigma_{\mathrm{z}}$ is computed

$$
\begin{aligned}
& \Sigma_{\mathrm{z}}=E\left\{\mathbf{z z}^{T}\right\}-E\{\mathbf{z}\} E\left\{\mathbf{z}^{T}\right\} \\
& =E\left\{\left(\boldsymbol{A} \boldsymbol{b}+\boldsymbol{R}^{-1} \boldsymbol{n}+\boldsymbol{R}^{-1} \boldsymbol{j}\right)\left(\boldsymbol{A} \boldsymbol{b}+\boldsymbol{R}^{-1} \boldsymbol{n}+\boldsymbol{R}^{-1} \boldsymbol{j}\right)^{T}\right\}-(\boldsymbol{A} \boldsymbol{b})(\boldsymbol{A} \boldsymbol{b})^{T} \\
& =E\left\{\boldsymbol{A} \boldsymbol{b}(\boldsymbol{A} \boldsymbol{b})^{T}+\boldsymbol{A} \boldsymbol{b}\left(\boldsymbol{R}^{-1} \boldsymbol{n}\right)^{T}+\boldsymbol{A} \boldsymbol{b}\left(\boldsymbol{R}^{-1} \boldsymbol{j}\right)^{T}+\boldsymbol{R}^{-1} \boldsymbol{n} \boldsymbol{b}^{T} \boldsymbol{A}^{T}+\boldsymbol{R}^{-1} \boldsymbol{n n} \boldsymbol{n}^{T}\left(\boldsymbol{R}^{-1}\right)^{T}+\right. \\
& \left.\boldsymbol{R}^{-1} \boldsymbol{n} \boldsymbol{j}^{T}\left(\boldsymbol{R}^{-1}\right)^{T}+\boldsymbol{R}^{-1} \boldsymbol{j} \boldsymbol{b}^{T} \boldsymbol{A}^{T}+\boldsymbol{R}^{-1} \boldsymbol{j} \boldsymbol{n}^{T}\left(\boldsymbol{R}^{-1}\right)^{T}+\boldsymbol{R}^{-1} \boldsymbol{j}^{T}\left(\boldsymbol{R}^{-1}\right)^{T}\right\}-(\boldsymbol{A} \boldsymbol{b})(\boldsymbol{A} \boldsymbol{b})^{T} \\
& =\boldsymbol{A} \boldsymbol{b} E\left\{\boldsymbol{n}^{T}\right\}\left(\boldsymbol{R}^{-1}\right)^{T}+\boldsymbol{A} \boldsymbol{b} E\left\{\boldsymbol{j}^{T}\right\}\left(\boldsymbol{R}^{-1}\right)^{T}+\boldsymbol{R}^{-1} E\{\boldsymbol{n}\} \boldsymbol{b}^{T} \boldsymbol{A}^{T}+\boldsymbol{R}^{-1} E\left\{\boldsymbol{n} \boldsymbol{n}^{T}\right\}\left(\boldsymbol{R}^{-1}\right)^{T} \\
& +\boldsymbol{R}^{-1} E\{\boldsymbol{n}\} E\left\{\boldsymbol{j}^{T}\right\}\left(\boldsymbol{R}^{-1}\right)^{T}+\boldsymbol{R}^{-1} E\{\boldsymbol{j}\} \boldsymbol{b}^{T} \boldsymbol{A}^{T}+\boldsymbol{R}^{-1} E\{\boldsymbol{j}\} E\left\{\boldsymbol{n}^{T}\right\}\left(\boldsymbol{R}^{-1}\right)^{T} \\
& +\boldsymbol{R}^{-1} E\left\{\boldsymbol{j j}^{T}\right\}\left(\boldsymbol{R}^{-1}\right)^{T} \\
& =\sigma_{n}^{2}\left(\boldsymbol{R}^{-1}\right)^{T}+\sigma_{j}^{2}\left(\boldsymbol{R}^{-1}\right)^{T}
\end{aligned}
$$

where $E\{\boldsymbol{n}\}=0$ and $E\left\{\boldsymbol{n} \boldsymbol{n}^{T}\right\}=\sigma_{n}^{2} \boldsymbol{R}$ and $\sigma_{n}^{2}$ is the power of the AWGN at the output of the matched filter, $E\{\boldsymbol{j}\}=0$ and $E\{\boldsymbol{j} \boldsymbol{j}\}=\sigma_{j}^{2} \boldsymbol{R}$ and $\sigma_{j}^{2}$ is the power of the partial band jamming at the output of the matched filter.

The probability of bit error of the $k^{\text {th }}$ user can be written as: 


$$
P_{e}=Q\left(\sqrt{\frac{E\left(z_{k} \mid b_{k}\right)^{2}}{\operatorname{var}(z)}}\right)=Q\left(\sqrt{\frac{\left(\sqrt{\frac{P}{2}} T_{b}\right)^{2}}{\left.\left(\frac{N_{O} T_{b}}{4}+\frac{N_{j} T_{b}}{4 \rho}\right)^{-1}\right)_{k, k}}}\right)=Q\left(\sqrt{\left(\frac{2 E_{b}}{N_{o}}+\frac{2 E_{b} \rho}{N_{j}}\right) \frac{1}{\left(R^{-1}\right)_{k, k}}}\right)
$$

where $\left(R^{-1}\right)_{k, k}$ is the value of inverted cross-correlation of the spreading code for the $k^{\text {th }}$ user.

\section{Numerical Results and Discussions}

The theoretical expressions derived in section III are validated under different conditions such as jamming in the presence of AWGN and MAI. A spreading factor that is corresponding to $\mathrm{N}=31$ is assumed to be a code for a signal to jamming ratio equal to $10 \mathrm{~dB}$.

In Figs. 2 and 3 the probability of error for SUMF and decorrelator receiver respectively are presented with different values of partial band jamming factor $\rho$. Thus it is clear that by decreasing $\rho$ the performance of both receivers degrades and for smaller values of $\rho$ the performance will dramatically degrade.

In Figs. 4 and 5 the probability of error for SUMF and decorrelator receiver respectively are presented against the number of users. It is clear that increasing the number of users affects the performance of SUMF much more than the decorrelator receiver that can obtain the same performance by increasing the number of users. The decorrelator began to severely degrades when it approaches the maximum number of allowed user; (according to the code length).

In Fig. 6 the probability of error for SUMF and decorrelator receivers are presented under partial band jamming factor $\rho=0.5$. It is clear that SUMF receiver has better performance than decorrelator that is because decorrelator receiver increases the noise power so it does not perform properly under partial band jamming.

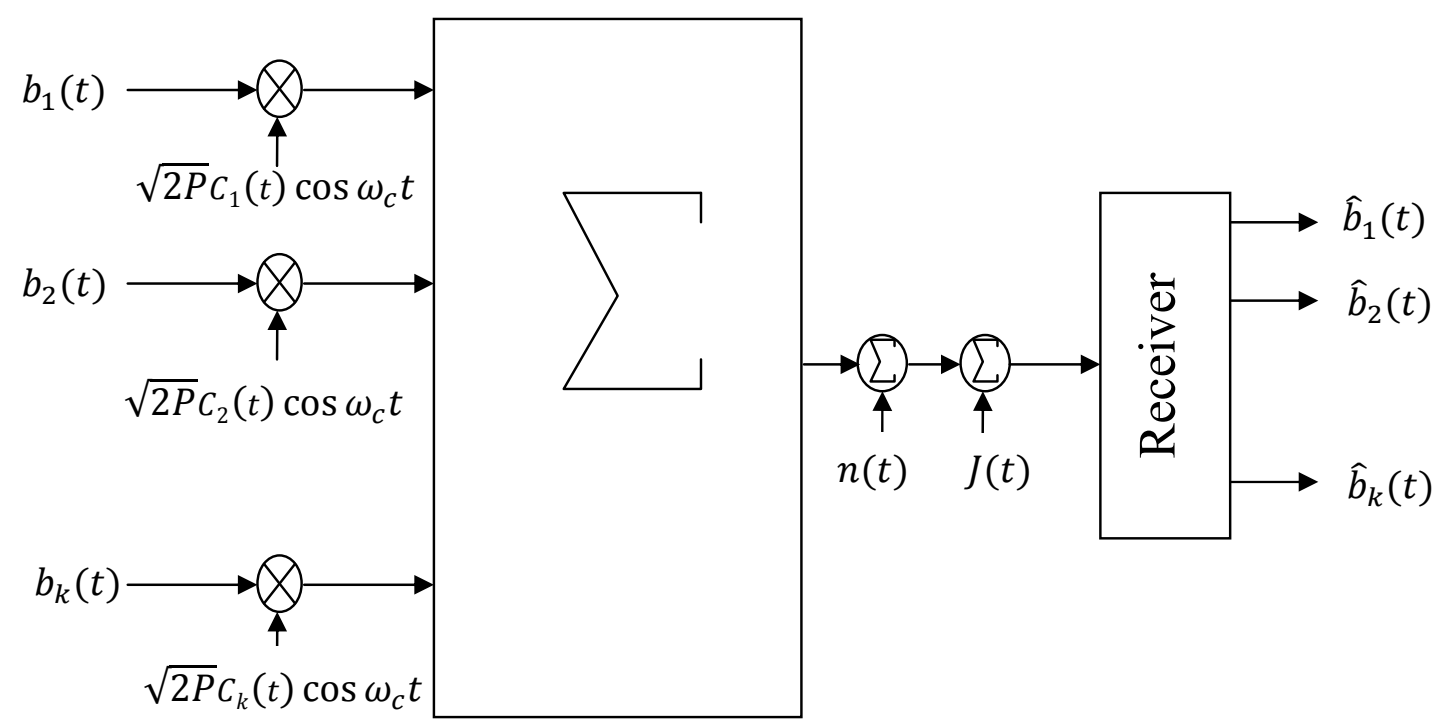

Fig. 1 The block diagram of a DS-CDMA system with jamming 


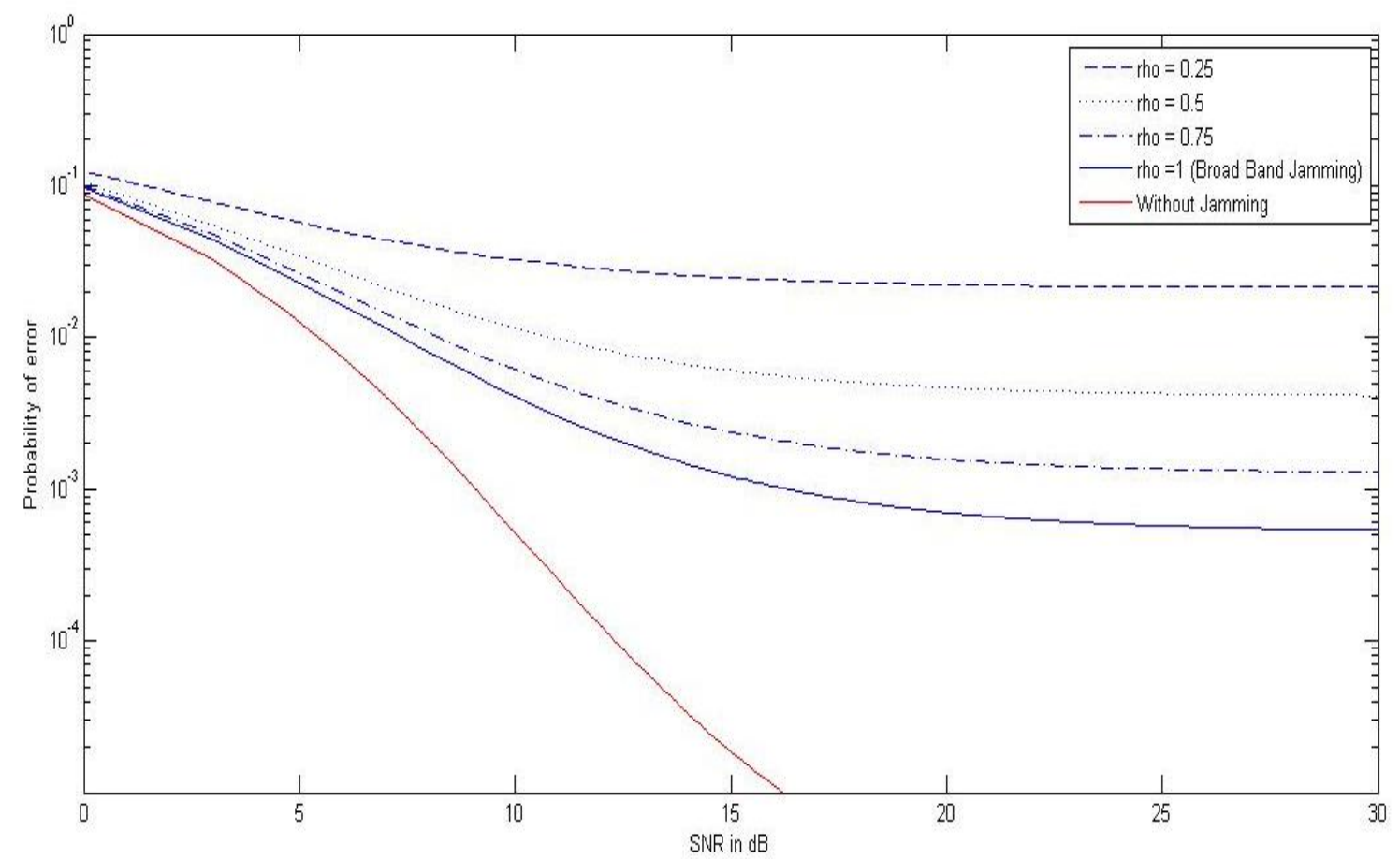

Fig. 2 Performance of SUMF receiver in presence of partial band jamming with $\rho=0.25,0.5,0.75$ and 1 .

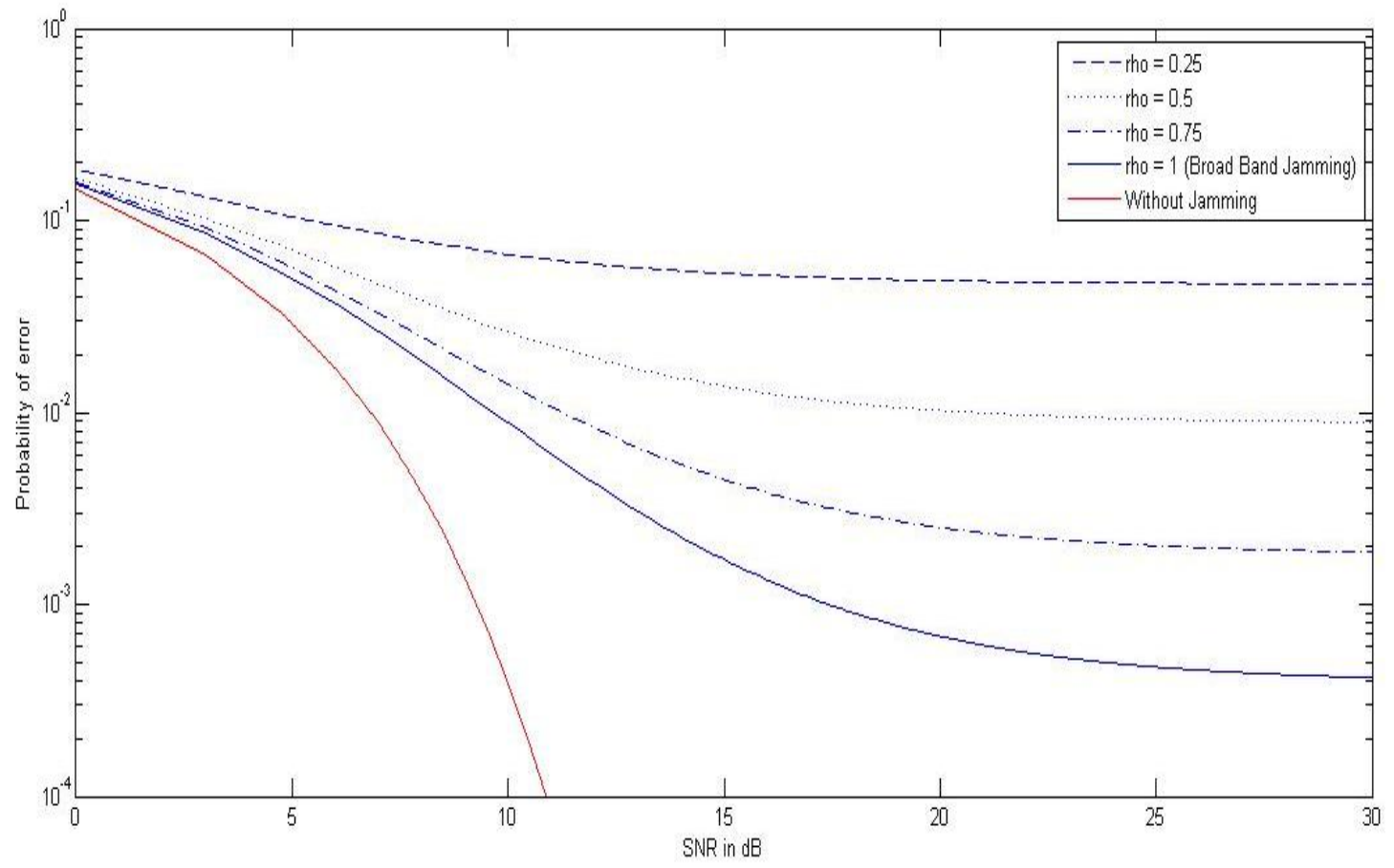

Fig. 3 Performance of decorrelator receiver in presence of partial band jamming with $\rho=0.25,0.5,0.75$ and 1 . 


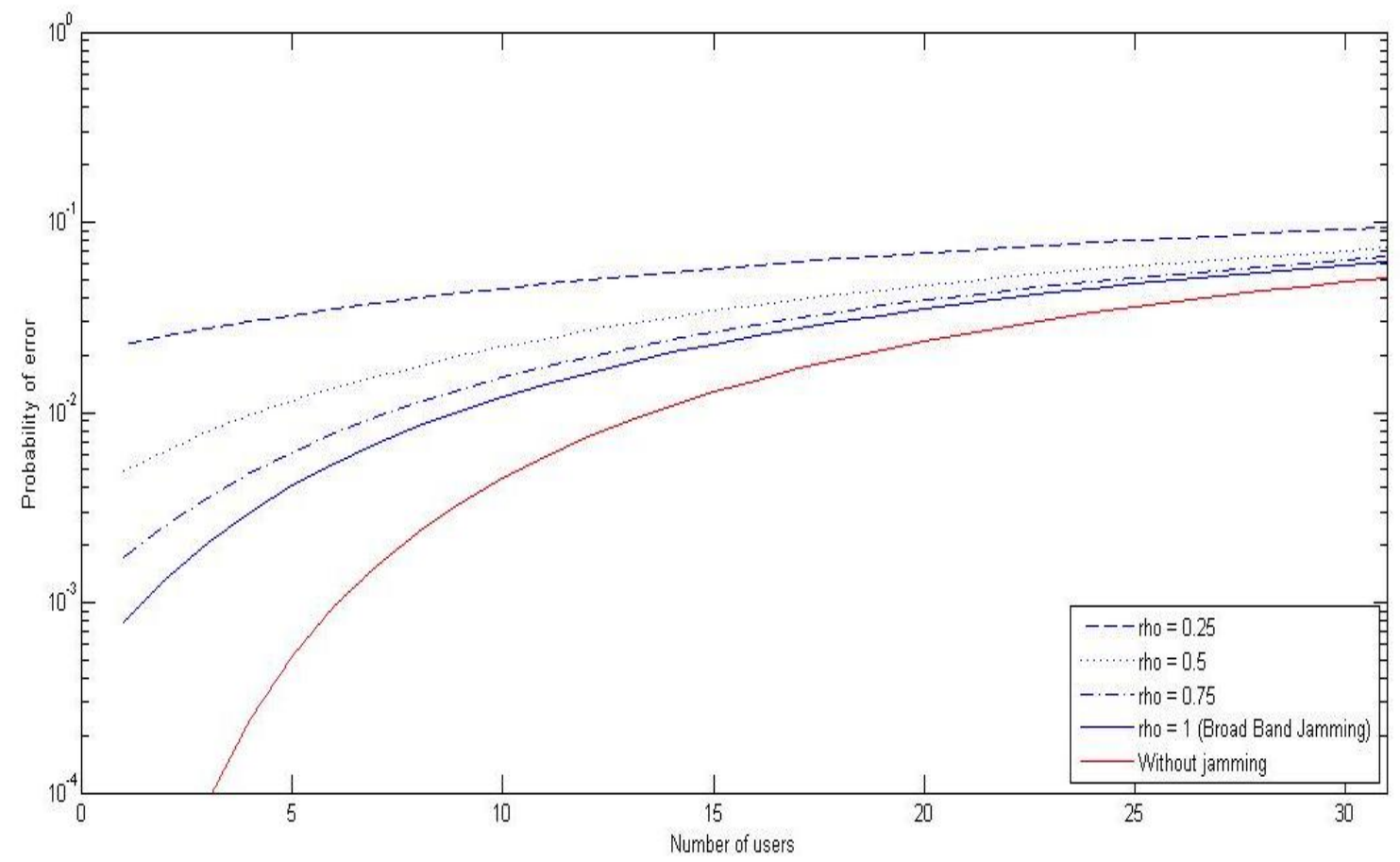

Fig. 4 Performance of SUMF receiver against number of users in presence of partial band jamming $\rho=0.25,0.5,0.75$ and 1 .

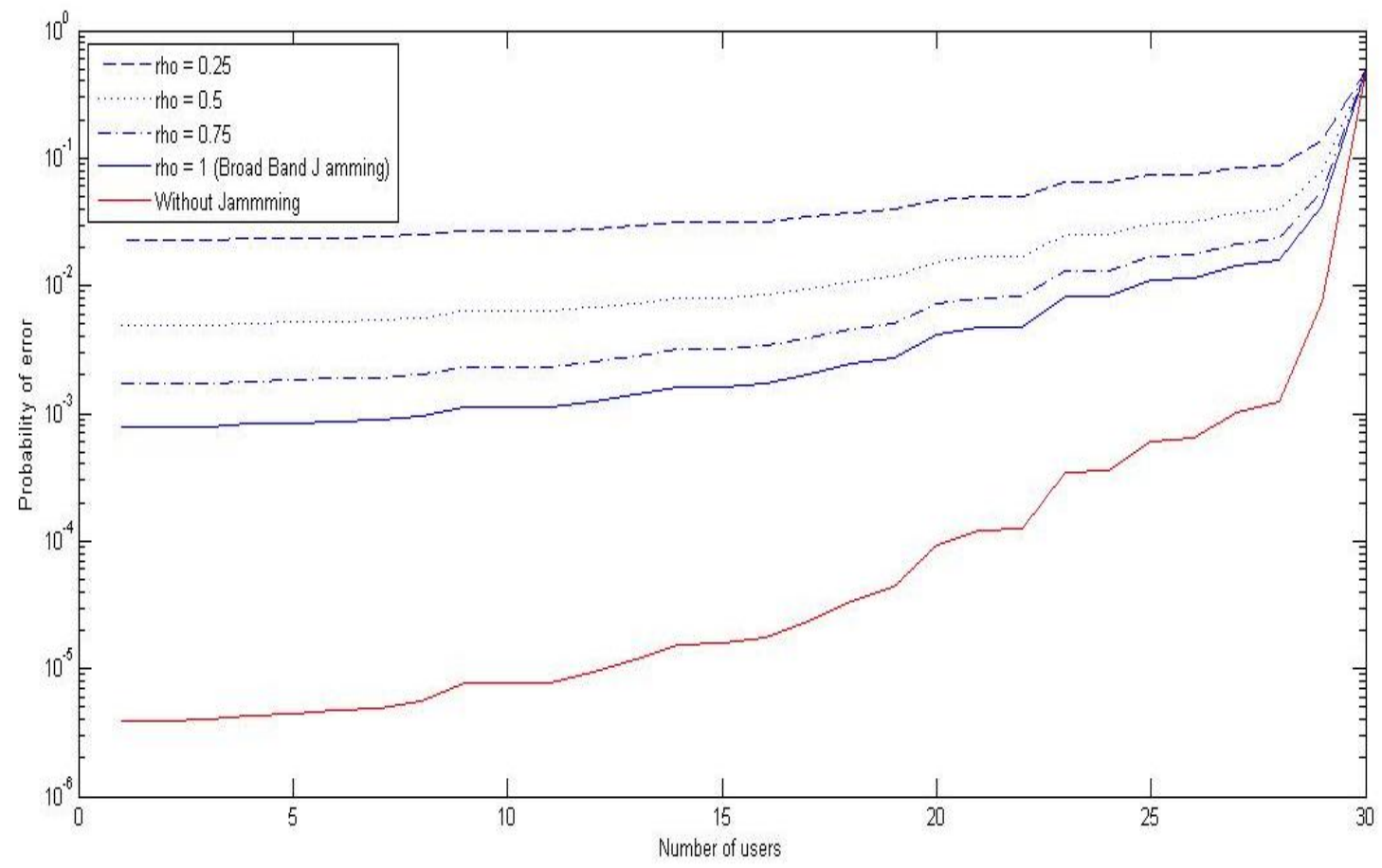

Fig. 5 Performance of decorrelator receiver against number of users in presence of partial band jamming $\rho=0.25,0.5,0.75$ and 1 . 


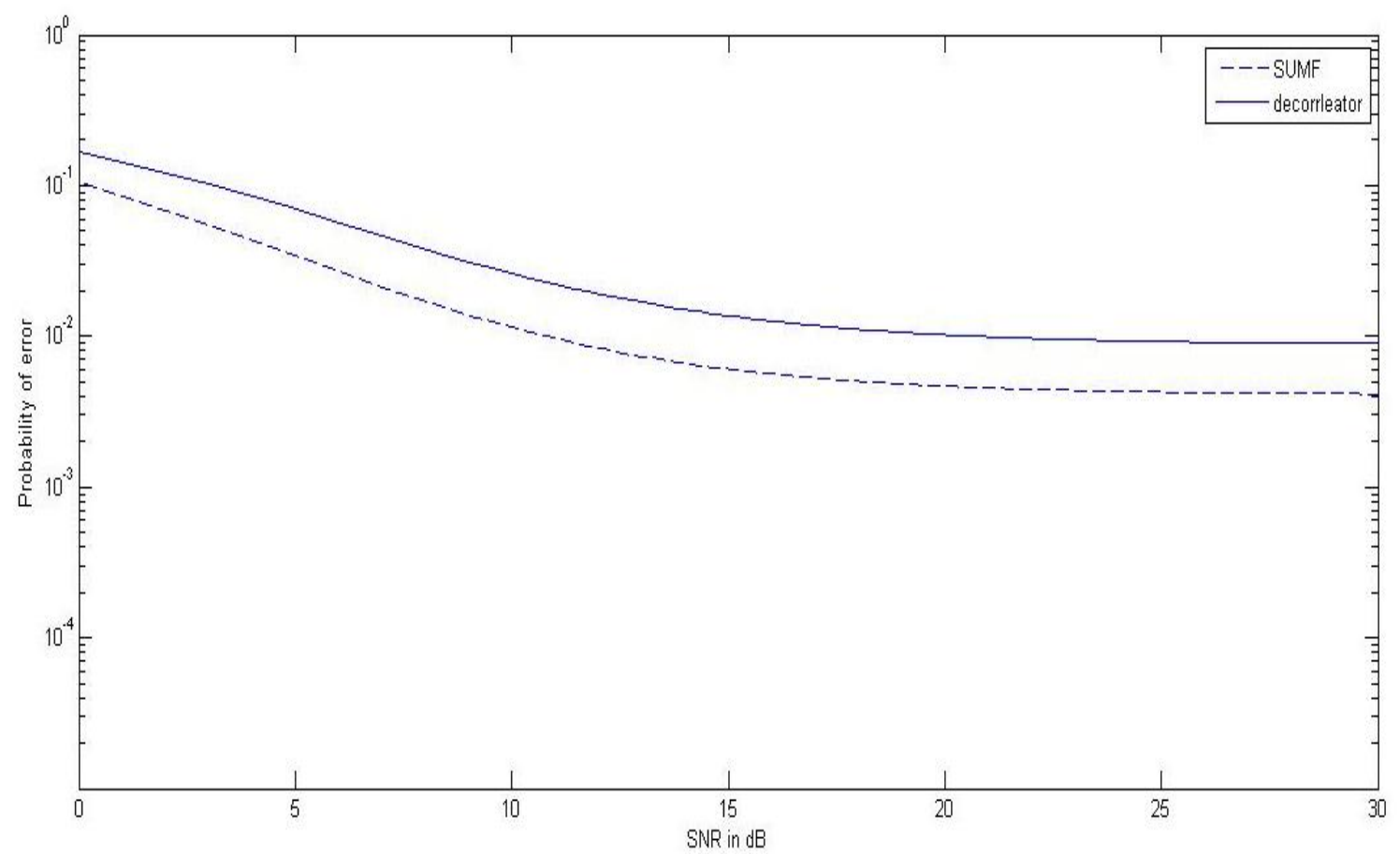

Fig. 6 Performance comparison of SUMF and decorrelator receivers.

\section{Conclusions}

The performance of SUMF and decorrelator receiver in presence of partial band jamming has been evaluated. It is shown that decreasing partial band jamming factor degrades the performance of both receivers, however the SUMF receiver has better performance relative to the decorrelator receiver. Moreover, increasing number of users affects the performance of the SUMF receiver much more than that of the decorrelator receiver.

\section{References}

[1] Khalifa Nasser K Jileta, Mahamod Ismail, Alina Marie Hasbi, "Performance of Multi tone Code Division Multiple Access (MT-CDMA) in an AWGN Channel and in Presence of Narrowband Jamming," IEEE 7th Malaysia International Conference on Communication, Nov. 2005.

[2] R F Ormondroyd, E Al-Susa, "Impact of Multipath Fading and Partial-Band Interference on the Performance of a COFDM/CDMA Modulation Scheme for Robust Wireless Communications," Military Communications Conference, MILCOM 98. Proceedings. Oct 1998, IEEE vol. 2, pp. 673-678, 18-21.

[3] R. Michael Buehrer, Code Division Multiple Access (CDMA), Morgan and Claypool 2006

[4] Theodore S. Rappaport, Wireless Communications, Prentic Hall PTR 2002

[5] L. B. Milstein, S. Davidovici and D. L. Schilling, "The effect of multiple-tone interfering signals on a direct sequence spread spectrum communication system," IEEE Trans.Commun, Mar.1982, vol. COM-30, pp. 436-446.

[6] N. Nazari and R. E. Ziemer, "Computationally efficient bounds for the performance of direct sequence spread spectrum multiple-acces communications systems in jamming environments, " IEEE Trans. Commun, May 1988, vol. 36, No. 5, pp. 577-587. 